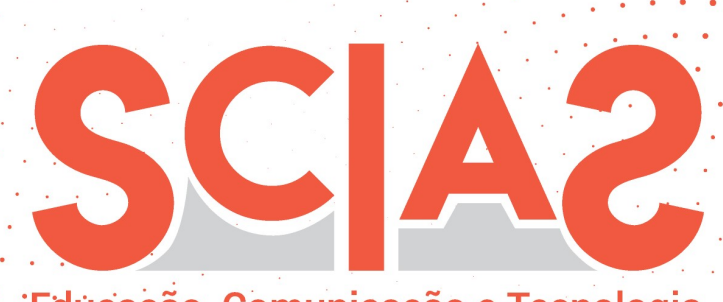

'Edúçaçã̃o, Comunicação e Tecnologia'

Atribuição BB CY 4.0

\title{
Conectando pessoas por meio de interesses compartilhados: avaliação da aprendizagem com o aplicativo QuizUp
}

\author{
Verissimo Barros dos Santos Junior ${ }^{1}$ \\ Jean Carlos da Silva Monteiro²
}

\section{Resumo}

Este estudo analisa as potencialidades do aplicativo QuizUp enquanto instrumento de avaliação da aprendizagem. Versa sobre um estudo descritivo e exploratório, que discute o app-learning na educação (aprendendo com aplicativos) e descreve o aplicativo QuizUp, apresentando as suas características, as suas funcionalidades e o seu principal objetivo, que é conectar pessoas por meio de interesses compartilhados. Verifica que na aplicação do quiz na sala de aula, os alunos podem interagir com usuários de todo o mundo em uma rede social, tornando-se um campo de debate sobre um determinado, fator que favorece a aprendizagem por meio da troca de informações e interação em âmbito global.

\section{Palavras-chave}

App-learning. Jogos na educação. Avaliação da aprendizagem. QuizUp.

Recebido em: 16/04/2020

Aprovado em: 21/07/2020

\footnotetext{
1 Especialista em Informática na Educação pelo Instituto Federal de Educação, Ciência e Tecnologia do Maranhão. Graduado em Administração pela Universidade Anhanguera. Professor dos cursos de Administração e Logística do Centro de Ensino Técnico e Profissionalizante do Maranhão - CEMP/MA.

Email: verissimo_barros@hotmail.com

2 Mestre em Cultura e Sociedade pela Universidade Federal do Maranhão. Especialista em Comunicação, Cultura e Tecnologia pela Faculdade Venda Nova do Imigrante. Graduado em Comunicação Social, habilitação em Jornalismo, pela Faculdade Estácio de São Luís.

Email: falecomjeanmonteiro@gmail.com
} 


\section{Connecting people through shared interests: Learning assessment with QuizUp app}

\section{Abstract}

This study analyses the potential of QuizUp app as a tool for learning assessment. It follows a descriptive-exploratory approach and aims at describing the QuizUp application in the educational context by presenting its characteristics, functionalities and main purpose, which is to connect people through shared interests. Results verified that the use of the app in the classroom allows students to interact with users from all over the world, in a social network model, being a fruitful field for emerging debates on relevant topics. In this sense, the app holds the power to enhance learning assessment through information exchange and interaction on a global level.

\section{Keywords}

App-learning. Education games. Learning Assessment. QuizUp. 


\section{Introdução}

Os alunos do século XXI fazem parte de uma Geração Conectada que mudou o seu jeito de perceber e interagir com o mundo ao seu redor devido à grande exposição às Tecnologias de Informação e Comunicação (SILVA; PINTO, 2019). Diante disso, as tecnologias digitais estão cada vez mais inseridas nos processos formativos, trazendo inúmeras contribuições para a aprendizagem.

Nesse cenário, surge o app-learning (uso de aplicativos na aprendizagem), termo cunhado por Couto, Porto e Santos (2016), em que os jogos podem ser utilizados como boas estratégias pedagógicas, ajudando a desenvolver habilidades e competências para o processo de formação e aprendizagem ao longo da vida.

Este estudo analisa as potencialidades do aplicativo QuizUp enquanto instrumento de avaliação da aprendizagem. Versa sobre um estudo descritivo e exploratório, que discute o app-learning na educação (aprendendo com aplicativos) e descreve o QuizUp, apresentando suas características, funcionalidades e seu principal objetivo, que é conectar pessoas por meio de interesses compartilhados.

\section{App-learning}

Em uma sociedade cada vez mais conectada, utiliza-se aplicativos com as diversas funcionalidades. Já que esses aplicativos estão ao alcance dos alunos, por que não utilizar para enriquecer o processo de aprendizagem? O applearning trata-se de um modelo de aprendizagem que utiliza aplicativos para fins educacionais (MONTEIRO, et al. 2018).

Sobre o uso de aplicativos em sala de aula, Couto, Porto e Santos (2016) destacam que há um aspecto desse processo interativo na aprendizagem que merece reflexão: o papel promissor dos professores que sabem transferir esses aplicativos para fins de aprendizagem colaborativa, divertida, flexível e eficaz. Em síntese, app-learning se transformou em um aliado ao processo de aprendizagem por tornar a sala de aula mais interativa, suscitando a 
interatividade e a motivação configuradas em um processo formativo mais adequado a uma geração de alunos conectados que convivem em diversos ambientes permeados de ferramentas digitais (SILVA; PINTO, 2019).

\section{Jogos na educação}

O emprego dos jogos na sala de aula permite ao professor ressignificar o processo de construção do conhecimento de forma mais interativa, lúdica e colaborativa, transformando o modelo de ensino tradicional que, muitas vezes, não atende mais às demandas de alunos cada vez mais conectadas (BOTTENTUIT JUNIOR, 2017).

Savi e Ulbricht (2018) apontam dois aspectos fundamentais que reforçam a importância dos jogos digitais no processo de aprendizagem:

- o efeito motivador, visto que os jogos mostram a grande capacidade que têm de divertir e entreter o público ao mesmo tempo em que estimulam o aprendizado por conta dos ambientes totalmente interativos, desafios, curiosidades e fantasias;

- o efeito facilitador, os jogos além de facilitarem a aquisição de conteúdos também auxiliam no desenvolvimento de outras habilidades importantes como resolução de problemas, tomada de decisão, pensamento estratégico, trabalho em grupo, socialização, coordenação motora, entre outros.

Os jogos digitais agregados ao processo de aprendizagem visam chamar a atenção dos alunos por meio de uma metodologia atrativa, lúdica, envolvente e desafiadora, abordando os conteúdos de forma diferente e corroborando para o desenvolvimento de diferentes habilidades (MATTAR, 2019).

\section{Quizup, o maior jogo multiplayer do mundo}

O QuizUp, conforme a Figura 1, é um aplicativo desenvolvido pela empresa Plain Vanilla Games, online e multiplayer que tem como atração a oferta de perguntas e respostas com questões de múltipla escolha, cronometradas ao 
longo da disputa. Os jogadores podem escolher um dos temas implantados em sua interface e começar a jogar (QUIZUP, 2020).

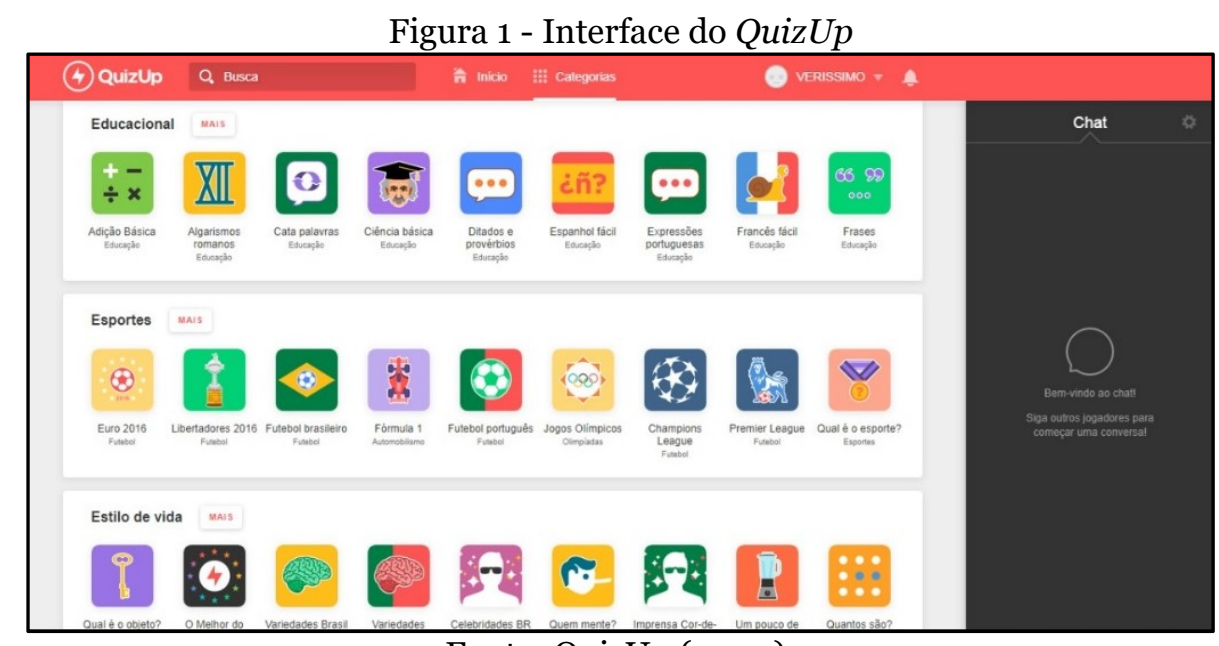

Fonte: QuizUp (2020).

São perguntas curtas e objetivas, trabalhando no usuário a rapidez e a precisão do conhecimento adquiridos por meio das aulas sobre um determinado assunto. Ao continuar jogando, há repetição de algumas perguntas, proporcionando ao usuário a absorção de conceitos básicos sobre o tema trabalhado, pela metodologia da reiteração.

Ao responder as perguntas de um quiz, o aplicativo disponibiliza ao usuário a alternativa correta, incentivando o mesmo a comparar o erro com o acerto. Além disso o QuizUp possibilita a participação ativa de seus usuários como criadores de novas perguntas, sendo estas compartilhadas ao público, por meio da autorização do responsável pelo jogo (QUIZUP, 2020).

Após cada partida, o usuário acumula um determinado número de pontos equivalente à quantidade de perguntas respondidas corretamente, exibido na Figura 2. 
Figura 2-Ranking no QuizUp

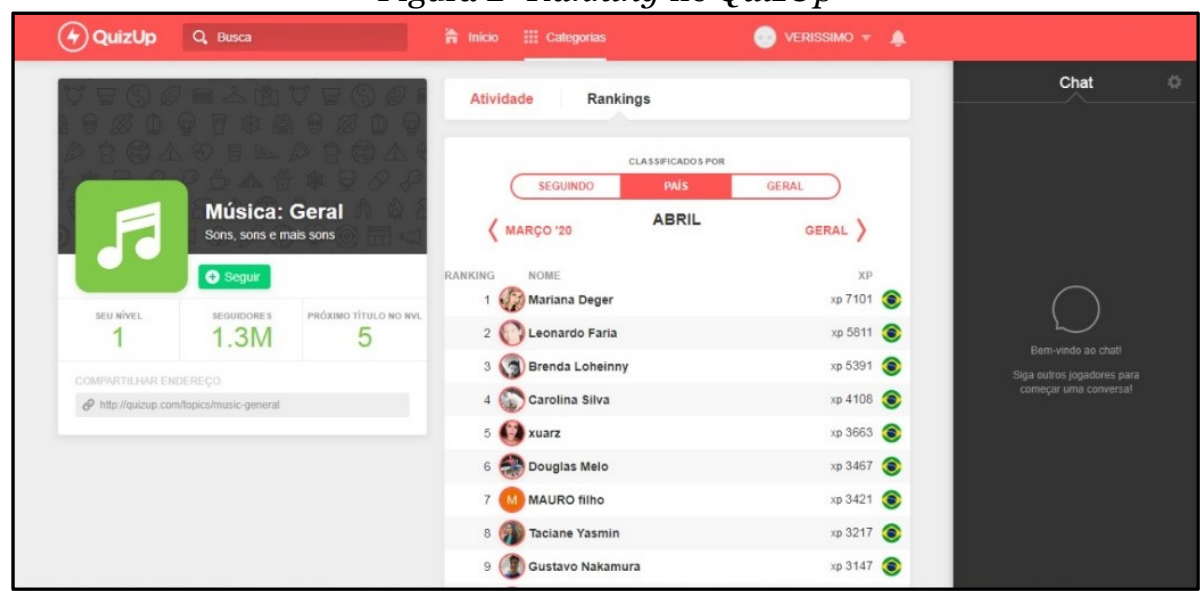

Fonte: QuizUp (2020).

Este número de pontos é adicionado a um ranking contendo os pontos dos outros participantes da mesma categoria, provocando, assim, uma certa competitividade entre os mesmos.

\section{Criando categorias no Quizup}

No aplicativo em análise, os participantes têm a opção de fazer login por meio de suas redes e mídias sociais digitais, como Facebook, Twitter ou Google+, que os permitem ver e seguir novos membros, convidar amigos para se juntar a eles ou desafiá-los a um jogo diante de uma temática de interesse dos dois (QUIZUP, 2020).

O professor tem a liberdade de criar novas categorias para a colaboração no jogo de forma livre ou manter as perguntas da categoria para um ciclo específico de pessoas por meio do compartilhamento de um link.

Pode-se utilizar essa ferramenta para realizar atividades na turma, tornando a sala de aula em um ambiente que viabiliza a interação do aluno e sua participação ativa no processo de aprendizagem. Neste caso, se configura como mediação ativa, como colabora Lorenzoni (2016):

Gamificação (ou, em inglês, gamification) tornou-se uma das apostas da educação no século 21. O termo significa simplesmente usar elementos dos jogos de forma a engajar pessoas para atingir um objetivo. Na educação, o potencial da gamificação é imenso: ela funciona para despertar interesse, aumentar a participação, desenvolver criatividade e autonomia, promover diálogo e resolver situações-problema. 
O primeiro passo para a criação das categorias é a identificação do nome, com até 25 caracteres para compor o título.Após a identificação, se faz necessário elaborar a descrição da sua categoria. Nessa área a criatividade tem vez e o ideal é utilizar uma linguagem clara e objetiva em até 45 caracteres - um resumo ou convite para as questões. Em seguida, o professor escolherá o idioma que deseja, apresentado na Figura 3.

Figura 3 - Criação das categorias no QuizUp

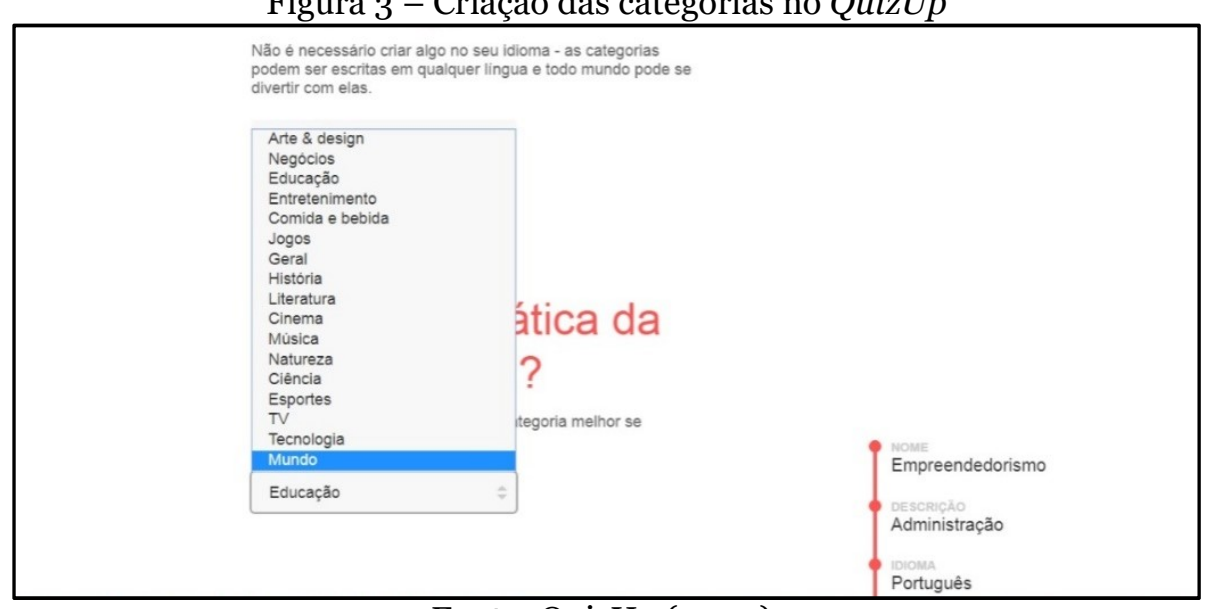

Fonte: QuizUp (2020).

A escolha da temática é a sequência da elaboração e pode ser escolhida dentre as 17 opções disponíveis pelo site: arte e design; negócios; educação; entretenimento; comida e bebida, jogos, geral, história; literatura; cinema; música; natureza; ciências; esportes; TV; tecnologia e mundo, como na Figura 4.

Figura 4 - Escolha da temáticano QuizUp

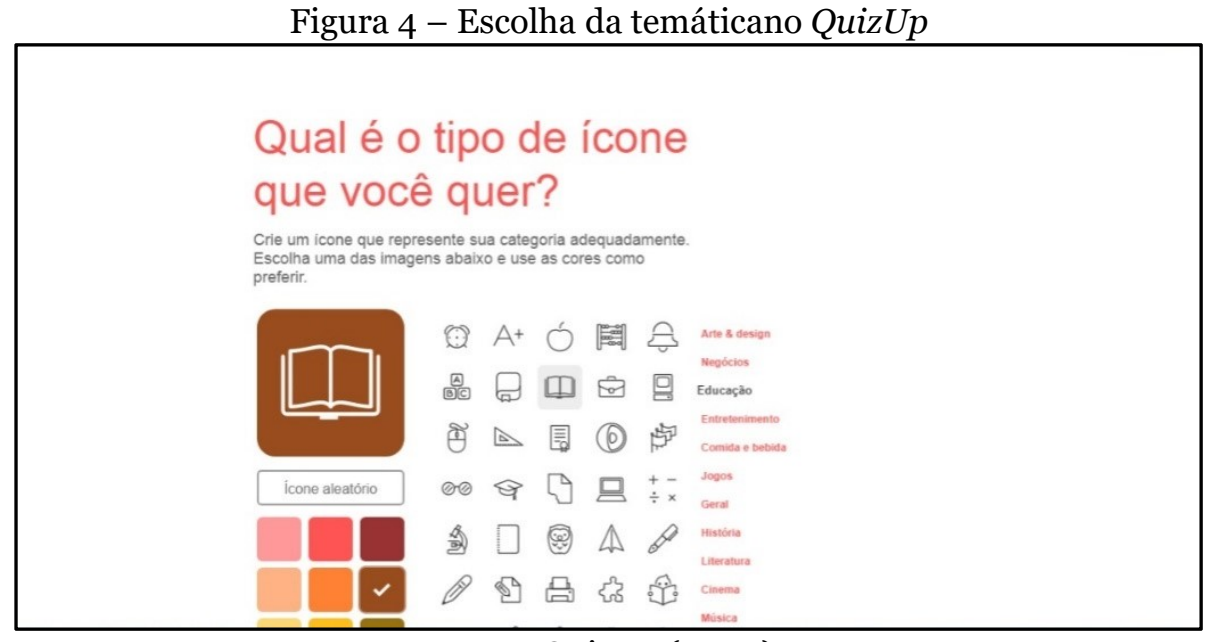

Fonte: QuizUp (2020). 
É necessário criar um ícone que representa a categoria do quiz adequadamente. O professor é convidado a escolher uma das imagens disponíveis e usar as cores como preferir. Pronto! Com toda a configuração concluída as questões podem ser criadas e incluídas no QuizUp. Todas as categorias precisam de algumas perguntas. Mesmo sendo a primeira a ser criadas e incluídas, as perguntas de cada jogo serão selecionadas aleatoriamente e não na ordem que são criadas e informadas pelo professor.

O enunciado das perguntas pode ser descrito em até 130 caracteres e as alternativas em até 30 caracteres, podendo ser utilizado quatro alternativas e somente uma correta, como indicado na Figura 5.

Figura 5 - Enunciado das perguntas no QuizUp

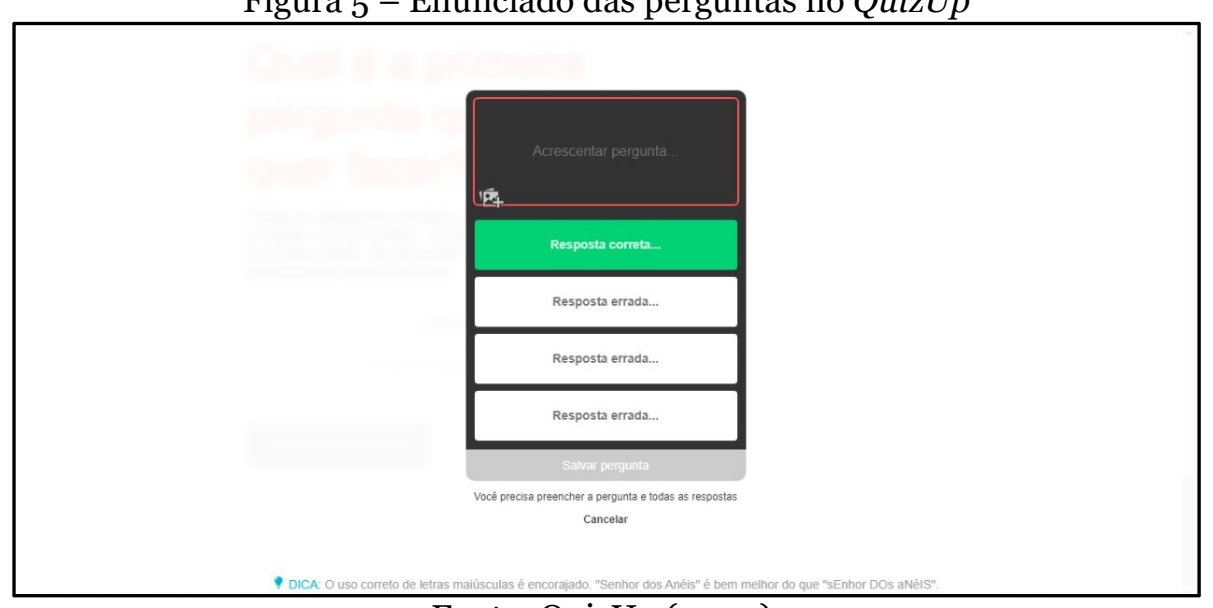

Fonte: QuizUp (2020).

Ao finalizar a elaboração das categorias, o professor pode disponibilizar o link aos alunos para participarem do quiz. A participação dos alunos nesse processo pode ser síncrona ou assíncrona.

\section{Avaliação da aprendizagem com o aplicativo Quizup}

$\mathrm{Na}$ avaliação de forma síncronas é necessária a participação do aluno e do professor no mesmo instante e no mesmo ambiente. Assim sendo, ambos devem se conectar no mesmo momento e interagir entre si. Os estudantes também podem surgir com alguma dúvida e apresentá-las ao professor em tempo real. 
O principal benefício da aplicação do quiz nesta modalidade é a praticidade para tirar dúvidas. Além disso, o professor pode trabalhar com os alunos a análise da questão e abrir para momentos de dúvidas. O que é uma grande vantagem para aumentar o engajamento dos estudantes, aspecto apontado nos estudos de Bottentuit Junior (2017) como um dos contributos da aplicação de jogos na educação.

Ao realizar o quiz na forma assíncronas não é necessário que os alunos e professores estejam conectados ao mesmo tempo para que as tarefas sejam concluídas e o aprendizado seja adequado. Dessa forma, o professor pode disponibilizar o link ao aluno participar do jogo respondendo das questões de casa, do trabalho ou de qualquer outro lugar com acesso à internet.

Nesse ponto de vista, Couto, Porto e Santos (2016) explicam que a mobilidade e à ubiquidade oferecidas pelo app-learning permite que as TIC se apresentem potencialmente para uma construção do conhecimento mais informal, proporcionando ambientes para uma aprendizagem mais flexível e menos centralizada no espaço físico da escola.

Sobre a interação de pessoas por interesse compartilhados, o aplicativo pode ser utilizado em todos os países e os alunos de todo o mundo podem escolher temas ou subtemas que são trabalhados durante a aula (categorias no aplicativo). A participação dos alunos no quiz ocorre dentro de grupo com características de rede social e dentro da proposta de interatividade apontados por Silva e Pinto (2019), no qual os eles podem compartilhar seus no feed as fotos, vídeos e textos.

Esse fator cria interação com a rede que se conecta por meio de um tema que agrada os determinados grupos, formados por interesses compartilhados. Ao analisar o comportamento de publicação em um dos grupos, percebe-se que as publicações nem sempre são favoráveis a aprendizagem dos alunos, favor que não agrega para a finalidade do objetivo da rede social. 
A utilização do QuizUp em sala de aula vão ao encontro dos estudos de Savi e Ulbricht (2018) e evidenciou-se algumas finalidades pedagógicas, em como disponibilizar um apoio didático interativo para a abordagem de diferentes temas relacionados ao conteúdo da disciplina; despertar o interesse do aluno pelo tema que será estudado; checar conhecimentos preexistentes; fornece mais informações sobre assuntos já trabalhados em sala de aula; incentivar pesquisas sobre temas discutidos em sala, indicando fontes confiáveis.

A teoria da utilização estratégica dos jogos, levantada por Mattar (2019), possibilitou também comprovar que o aplicativo também favorece a fixação do conteúdo estudado, pois, quanto mais partidas o usuário joga, mais vezes ele responde às mesmas perguntas do tema e, a cada partida, pode obter mais respostas corretas.

Considerando que a competitividade, de maneira geral, cria um certo estímulo ao ser humano de continuar uma certa atividade. E, diante disso, pode-se concluir que é um fator determinante quando aplicada ao aprendizado.

\section{Considerações finais}

Os jogos digitais são importantes recursos para o ensino uma vez que permitem associar conteúdo diversos ao entretenimento e amotivação, fator importante no processo de aprendizagem.Ao analisar as características do aplicativo QuizUp, sobretudo com a sua utilização em sala de aula, percebe-se que favorece o estímulo da competitividade saudável entre os alunos, transformam a sala de aula em um ambiente ainda mais produtivo para um maior coeficiente de rendimento dos alunos.

Com a inserção dos smartphones e da conectividade, os jovens realizam praticamente todas suas tarefas por meio de aplicativos e mediante a este novo cenário, a necessidade dos professores se adequarem a estas tecnologias e utilizarem como meio de disseminar e fixar o conteúdo para os seus alunos. 
O QuizUp em sala de aula favorece o engajamento de seus participantes, a competitividade saudável e a motivação para estar sempre atento ao conteúdo transmitido. O aplicativo se apresenta como instrumento de auxílio para as atividades do professor, porque ele obtém o resultado das atividades por meio de um ranking da turma.

Por fim, a aplicação do QuizUp em modalidades assíncronas demanda dos alunos um foco maior para que as suas publicações na rede social sejam feitas para fins pedagógicos e do professor para continuar motivando os alunos a interagirem nos comentários das postagens a fim de criar discussões sobre o tema abordado.

\section{Referências}

BOTTENTUIT JUNIOR, J. B. O Aplicativo Kahoot na Educação: verificando os conhecimentos dos alunos em tempo real. In: Maria João Gomes; Antonio José Osório; Antonio Luis Valente. (Org.). Challenges 2017: Aprender nas Nuvens, Learning in the Clouds. 15 ed. Braga - Portugal: Universidade do Minho UMINHO, 2017, v.1 p. 1587-1602.

COUTO, E.; PORTO, C.; SANTOS, E. (Org.). App-learning: experiências de pesquisa e formação. Salvador: EDUFBA, 2016.Gamificação: o que é e como pode transformar a aprendizagem. Disponível em: <https://www.geekie.com.br/blog/gamificacao/>. Acesso em Mar. 2020.

MATTAR, J. Games em educação: como os nativos digitais aprendem. São Paulo: Pearson Prentice Hall, 2010. 181 p.

MONTEIRO, J. C. S et al. Sociedade da aprendizagem: da ubiquidade aos novos paradigmas do app-learning. Revista Tecnologias na Educação, v. 27, p. 1$13,2018$.

QUIZUP. The Biggest Trivia Game in the World. [S.l.: s.n.], 2020. Disponível em: <https://www.quizup.com/>. Acesso em: 09 mar. 2020.

SAVI, R; ULBRICHT, V. R. Jogos Digitais Educacionais: Benefícios e Desafios. RENOTE. Revista Novas Tecnologias na Educação, v. 6, p. 1-10, 2018.

SILVA, J.; PINTO, A. Geração C: Conectados em novos modelos de aprendizagem. Rio de Janeiro: VIII Brazilian Symposium on Games and Digital Entertainment, 2019. 